in Cell and Developmental Biology

Elsevier Editorial System(tm) for Seminars

\author{
Manuscript Draft
}

Manuscript Number: YSCDB-D-16-00134R2

Title: PARL: the mitochondrial rhomboid protease

Article Type: SI: Rhomboid proteases in health \& dis.

Keywords: PARL; rhomboid; mitochondria; protease; cell death; metabolism

Corresponding Author: Prof. Bart De Strooper, Ph.D., MD

Corresponding Author's Institution: VIB

First Author: Marco Spinazzi, MD, PhD

Order of Authors: Marco Spinazzi, MD, PhD; Bart De Strooper, Ph.D., MD

Manuscript Region of Origin: BELGIUM

Abstract: The rhomboid family comprises evolutionary conserved

intramembrane proteases involved in a wide spectrum of biologically relevant activities. A mitochondrion-localized rhomboid, called PARL in mammals, and conserved in yeast and Drosophila as RBD1/PCP1 and rho-7, respectively, plays an indispensable role in cell homeostasis as illustrated by the severe phenotypes caused by its genetic ablation in the various investigated species. Although several substrates of PARL have been proposed to explain these phenotypes, there remains a lot of controversy in this important area of research. We review here the putative functions and substrates of PARL and its orthologues in different species, highlighting areas of uncertainty, and discuss its potential involvement in some prevalent diseases such as type II diabetes and Parkinson's disease. 
Leuven, 18.07.2016

To: Dr. John Davey, PhD DSc

Editor-in-Chief, Seminars in Cell and Developmental Biology

Dear John and Sin,

We hereby enclose a revised version of the manuscript "PARL: the mitochondrial rhomboid protease" for being considered for publication in your journal Seminars in Cell and Developmental Biology.

We modified the highlights as suggested. We apologize if the graphics of the scientific illustration is not outstanding but we have limited dedicated softwares and no specific expertize. Please feel free to improve it in accordance to the journal standards if this is found convenient. We sincerely thank you for this kind invitation and hope it may be suitable for publication.

Looking forward hearing from you,

With my Best Regards,

Bart and Marco 
Revision Notes

\section{Revision notes}

We modified as suggested the sentence in both the highlights and in the abstract: "Genetic deletion of mitochondrial rhomboid leads to severe phenotypes in different species" instead of lethal phenotypes. 


\section{Highlights}

- Mitochondrial rhomboids are evolutionary conserved intramembrane proteases with fundamental, although still incompletely understood, roles in cell homeostasis

- Genetic deletion of mitochondrial rhomboid leads to severe phenotypes in different species

- Knowledge of PARL substrates and physiological function is dynamically evolving, and might uncover differentiations among species

- PARL could play a role in the biology of disease of diabetes and Parkinson's disease 


\title{
PARL: the mitochondrial rhomboid protease
}

\author{
Marco Spinazzi ${ }^{\mathrm{a}}$, and Bart De Strooper ${ }^{\mathrm{a}, \mathrm{b}, \mathrm{c}}$ \\ ${ }^{\text {a }}$ VIB Center for the Biology of Disease, O\&N4 Herestraat 49 box 602, 3000 Leuven, Belgium \\ ${ }^{\mathrm{b}}$ KU Leuven Center for Human Genetics, O\&N4 Herestraat 49 box 602, 3000 Leuven, Belgium \\ ${ }^{c}$ UCL Institute of Neurology, University College London, WC1N 3BG, UK
}

Corresponding author at: VIB Center for the Biology of Disease, O\&N4 Herestraat 49 box 602 , 3000 Leuven, Belgium. Email address: bart.destrooper@cme.vib-kuleuven.be

\begin{abstract}
The rhomboid family comprises evolutionary conserved intramembrane proteases involved in a wide spectrum of biologically relevant activities. A mitochondrion-localized rhomboid, called PARL in mammals, and conserved in yeast and Drosophila as RBD1/PCP1 and rho-7, respectively, plays an indispensable role in cell homeostasis as illustrated by the severe phenotypes caused by its genetic ablation in the various investigated species. Although several substrates of PARL have been proposed to explain these phenotypes, there remains a lot of controversy in this important area of research. We review here the putative functions and substrates of PARL and its orthologues in different species, highlighting areas of uncertainty, and discuss its potential involvement in some prevalent diseases such as type II diabetes and Parkinson's disease.
\end{abstract}

\section{Keywords}

PARL, rhomboid, mitochondria, protease, cell death, metabolism 


\section{Contents}

1. Introduction

2. Phenotypes caused by genetic manipulations of the mitochondrial rhomboid in different organisms

2.1 The yeast mitochondrial rhomboid: Rbd1p/Pcp1p

2.2 The fly mitochondrial rhomboid: Rhomboid-7

2.3 Parl in zebrafish

2.4 Mammalian PARL

3. PARL substrates

3.1 OPA1

3.2 HTRA2/OMI

3.3 PINK1

\subsection{PGAM5}

4. Putative functions of PARL

4.1 A role of PARL in apoptosis

4.2 PARL in mitochondrial shape regulation

4.3 PARL mitochondrial function and homeostasis

4.4 PARL in mitophagy

5. A possible role of PARL in diabetes and Parkinson's Disease

6. Conclusions

References 


\section{Introduction}

The Presenilin associated rhomboid like (PARL) protease plays a pivotal but not entirely clarified role in the quality control and steady state maintenance of mitochondria. PARL was originally identified in an interaction screen aimed at elucidating the role of Presenilin 2 in apoptosis [1], which explains its name. Afterwards however it became clear that PARL was a mitochondrial resident protein [2], and that the link to presenilin was an artifact. Although it would have been more correct to redefine the protein name as "mitochondrial rhomboid," the name PARL was maintained for historical reasons. We suggest here that the meaning of the acronym PARL should be changed to "PGAM5/PINK1 associated rhomboid like", maintaining the historical gene name PARL to avoid confusion but providing a more correct association to its two identified substrates instead of linking it incorrectly to another intramembrane cleaving protease, presenilin. PARL is a member of the rhomboid family of intramembrane serine proteases and is composed of a core of six transmembrane helices with an additional transmembrane helix appended at the $\mathrm{N}$-terminus [3]. Conserved serine, histidine and asparagine catalytic residues, that are contributed by different transmembrane helices, provide the basis for the intramembrane proteolytic activity of PARL [4]. In addition to the catalytic domain, PARL holds an N-terminal vertebrate-specific $\mathrm{P} \beta$ domain. Although the physiological function of the $\mathrm{P} \beta$ domain is unknown, its biological relevance is emphasized by strong sequence conservation in mammals [3]. In the current review we focus on the biology of PARL, its different putative substrates and the question to what extent PARL is involved in type II diabetes and Parkinson Disease (PD). We start with discussing the loss of function phenotypes described in different organisms as those provide a first insight in the biological relevance of this unique protease.

\section{Phenotypes caused by genetic manipulations of the mitochondrial rhomboid in different organisms}

\subsection{The yeast mitochondrial rhomboid: Rbd1p/Pcp1p}

Rbd1p for rhomboid-1 or Pcp1p for "processing of cytochrome c peroxidase protein 1" is one of the two rhomboids in yeast. The second name derives from its first identified substrate, cytochrome c peroxidase, a ROS detoxifying enzyme [5]. For clarity and respecting historical nomenclature, we call this yeast rhomboid here Rbd1/Pcp1p and the encoding gene RBD1/PCP1. While following studies [6,7] convincingly demonstrated that cytochrome c peroxidase 1 is a genuine substrate of Pcplp, it became also clear that lack of cytochrome c peroxidase 1 had little visible consequences in yeast cells. Rbd1/Pcp1p deficient yeast strains are unable to grow in glycerol medium [2] and display striking mitochondrial morphological abnormalities, such as mitochondrial fragmentation and aggregation, as well as loss of mitochondrial DNA nucleoids [2]. These observations suggested an unprecedented fundamental role of this rhomboid in mitochondrial biology. 
An important clue came from the observation that the mitochondrial phenotype closely resembled that seen in Mgm1p deficient strains. Mgm1p, the yeast homolog of mammalian OPA1, is a dynamin-like GTPase involved in mitochondrial inner membrane fusion and mitochondrial DNA maintenance. Yeasts lacking Rbd1/Pcp1p are defective in Mgm1p processing, leading to increased levels of the full-length protein and reduced levels of its lower molecular weight form lacking the transmembrane domain, which anchors the protein to the inner mitochondrial membrane [2,8]. Expression of short Mgm1p could partially complement the $\Delta r b d 1$ metabolic phenotype, and expression of Rbd1/Pcp1p, but also of the human homolog PARL, restored the impaired processing of Mgm1p in the cells, while no rescue was seen with catalytically inactive mutants of Rbd1/Pcp1p or human PARL. This demonstrated that the cellular phenotypes and the absence of processing of Mgm1p were indeed caused by defective proteolytic function of the rhomboid, and not by some additional unknown function of this protease.

\subsection{The fly mitochondrial rhomboid: Rhomboid-7}

Rhomboid-7 (rho-7) deficient flies die mostly before pupation [9]. The few individuals that make it to adults suffer from neurological defects and male sterility, and do not survive longer than three days. Testis and skeletal muscle mitochondria from rhomboid-7 flies display morphological alterations suggestive of defective mitochondrial fusion. The flies also have disturbed abnormal electroretinograms (ERG) and displayed photoreceptor degeneration after prolonged light stimulation.

Similarly to rhomboid 1 in yeast, its orthologue Rhomboid-7 in Drosophila plays a critical role in mitochondrial membrane morphology regulation [9]. Down regulation of rhomboid-7 using double-stranded RNA in Drosophila S2 cells leads to severe fragmentation of the mitochondrial network, and again, similar phenotypes are seen when opal-like, the Drosophila orthologue of yeast $M G M 1$, is down regulated [9]. Intriguingly, overexpression of Rhomboid-7 led also to increased lethality and neurological deficits in Drosophila [10] and to a similar pathological mitochondrial aggregation as that seen by overexpressing PARL [9], but not catalytically inactive PARL. Thus, both overexpression and down regulation of Rhomboid-7 cause neurological phenotypes in vivo and morphological alterations in mitochondria implying that the proteolytic activity of this rhomboid has to be kept in check.

Interestingly, in the Rhomboid-7 overexpressing fly [10], the shorter isoform of Opa1-like was similarly expressed as in the wild-type, but absent in Rhomboid-7 deficient flies, while the amount of full length Opa1-like was paradoxically increased. Surprisingly, the Rhomboid-7 overexpression phenotype could then be rescued by introducing a heterozygous mutation of opal-like [10], suggesting that Rhomboid-7 and Opa1-like function in a common molecular pathway affecting mitochondrial integrity.

Overall, some but not all data are suggesting that Opa1-like is processed by Rhomboid 7, an hypothesis which could also explain the repetitively confirmed genetic interactions between rhomboid7/opal-like in Drosophila, RBD1/MGM1 in yeast, and Parl/Opal in mouse [2,9-11]. However, the processing of Opa1-like or OPA1 by Rhomboid-7 or PARL, respectively, has not 
been demonstrated directly by adding purified protease and substrate together. There is also lack of consistency in the different other experiments analyzing processing of Opa1-like and OPA1 in different assays and the evidence that OPA1 is mostly processed by other mitochondrial proteases, makes it a controversial issue whether OPA1 is an authentic substrate of PARL or not, as we will see below in our further discussion.

A medical important link between Rhomboid-7 and a pathway involved in the pathogenesis of Parkinson's Disease was made in Drosophila first. Mutations in human PINK1 and PARKIN genes are known to cause autosomal recessive Parkinson's disease. Genetic interactions and biochemical assays have also shown that pinkl and parkin act in the same pathway controlling mitophagy in Drosophila [12,13]. Interestingly, Pink1 overexpression enhances, and Pink1 inactivation decreases neurodegeneration in the eye ("rough eyes") caused by Rhomboid-7 overexpression [14]. The Pink1 overexpression phenotype is also characterized by "rough eyes" and it is partially rescued by ablation of parkin, but not by rhomboid-7. This places rhomboid-7 upstream of pinkl. Pinkl is in its turn upstream of parkin as shown previously [14], defining as such an important mitophagy pathway, which is believed to contribute to (inherited) forms of Parkinson's disease. Furthermore, crossing a 9myc-tagged pink1 transgenic line with the rhomboid-7 deficient flies, showed decreased processing of Pink1 thereby providing the first evidence that Pink1 is a substrate of the mitochondrial rhomboid [14]. Intriguingly, in the same study also a link with the mitochondrial serine protease HtrA2/Omi was made. As we will discuss below, full-length $\mathrm{HtrA} 2$ is inserted in the inner mitochondrial membrane but it becomes processed towards a soluble product. This soluble product was decreased in rhomboid-7 flies suggesting that HtrA2, like Pink1, is a substrate of this rhomboid [14]. Intriguingly, in this paper no effect on Opa1-like processing was observed, indicating that Rhomboid-7 was dispensable for processing of this substrate, despite the reported genetic interaction in other phenotypes [9,10]].

Altogether, the data in Drosophila indicate that Rhomboid-7 is an important mitochondrial enzyme implicated in Pink1, and possibly HtrA2 cleavage and biology. Processing of Opa1-like remains controversial. Follow-up is needed to have a more definitive answer to the question under which experimental conditions processing of these substrates occurs, whether there are other substrates still to be identified, and how processing of these substrates contributes to the severe phenotype in the fly. Also an assay to proof that Rhomboid-7 directly cleaves the proposed substrates is needed.

\subsection{Parl in zebrafish}

Two PARL orthologues have been identified in the zebrafish: parla and parlb [15]. Both parl transcripts are expressed during embryonic development and in adult tissues. Morpholinomediated knockdown of parla and parlb simultaneously causes embryonic lethality, increased acridine orange staining, and altered expression of tyrosin hydroxylase mRNA and dopamine transporter [15], which were used as markers of dopaminergic neurons. Interestingly, overexpression of zebrafish pinkl, but not kinase dead pinkl or pinkl containing Parkinson's 
disease causing mutations, rescues larval lethality or dopaminergic neurons abnormalities, indicating that parl is genetically upstream of pinkl, confirming this pathway in vertebrates.

\subsection{Mammalian PARL}

$\mathrm{Parl}^{-{ }_{-}^{-}}$mice develop normally during embryogenesis but start to suffer from a progressive multisystem disease a few weeks after birth. This wasting disease starts within a narrow time window in adulthood and is characterized by progressive muscle and immune organ atrophy, with death ultimately occurring before the age of three months [11]. Massive apoptosis was observed in thymus and spleen from 8 weeks-old animals, and double positive CD4+/CD8+ thymocytes were drastically reduced. Interestingly, major mitochondrial dysfunction and morphological abnormalities were not found in primary cell cultures derived from these mice. These observations contrast remarkably with the situation in yeast and Drosophila and might suggest that in mammalian cells mechanism are in place that at least partially compensate for the role of PARL in the control of mitochondrial membrane dynamics. Further study of the mitochondria of fibroblasts and liver from the Parl knock out animals during the induction of apoptosis showed more subtle phenotypes related to apoptosis susceptibility suggesting that Parl exerts anti-apoptotic activity. Isolated mitochondria from liver show faster remodeling of the cristae and release of cytochrome c upon stimulation of apoptosis, suggesting a role of Parl in the morphological maintenance of cristae during the execution of induced intrinsic apoptosis. Remarkably, this phenotype could be rescued by a soluble form of OPA1 but not by membrane bound OPA1, indicating the conservation of the Parl/Opa1 interaction from yeast to mice. Again, no direct evidence that PARL cleaves OPA1 could be provided, largely because a cell free system to measure PARL proteolytic activity has not been established yet.

It remains puzzling why in Drosophila and yeast dramatic morphological phenotypes in mitochondria are seen when the respective PARL homologues are inactivated, while in the Parl knock out MEFs and myotubes the mitochondrial network did not look altered and mitochondrial fusion rates were unchanged [11]. An important open question is whether the mitochondrial ultrastructure might be affected in cells and tissues from the $\mathrm{Parl}^{-/}$mouse at steady states. It remains also to be further explored to what extent the increased apoptosis rate in these animals can fully explain the progressive wasting phenotype.

\section{PARL substrates}

The further understanding of the molecular functions of PARL depends crucially on the unequivocal identification of its substrates. It is also important to take into account that different substrates might be cleaved in different physiological conditions and in different cells or tissues, something which might explain some of the controversy in the field. As we indicated already several candidate substrates have been identified, but the evidence remains largely indirect, and large knowledge gaps in the physiological and molecular function of PARL have become apparent. 


\subsection{OPA1}

OPA1 is considered a substrate of mammalian PARL by analogy with the yeast system [2]. Further work has shown that overexpression of a soluble OPA1 fragment, but not the full length (membrane bound) OPA1 rescues the susceptibility to induced apoptosis of the Parl knockout fibroblasts [11]. Indeed cell death is normalized and the faster release of cytochrome c seen in Parl deficient fibroblasts is counteracted by soluble OPA1. As discussed above, such genetic interaction was also seen in Drosophila and yeast, indicating a conserved pathway and linking OPA1 and PARL functionally to each other. Further evidence for such interaction comes from initial biochemical studies showing that OPA1 and PARL interact in yeast two hybrid screenings and can be co-immunoprecipitated from cells [11].

However, several other studies have indicated that PARL is dispensable for OPA1 processing [16]. Additional proteases are involved in the maturation of the different OPA1 forms, such as isoenzymes of the ATP-dependent matrix AAA proteases paraplegin [16], AFG3L1 and 2 [16-18], the inner mitochondrial protease OMA1 [18], and YME1L1 [19,20]. Therefore, while the genetic interaction between PARL and OPA1 seems to be established, the question remains open whether OPA1 is indeed a substrate of PARL. Possibly PARL cleaves OPA1 only under specific physiological or pathophysiological conditions. Another possibility remains that OPA1 and PARL interact in a non-enzymatic way.

\subsection{HTRA2/OMI}

The HtrA family comprises a group of serine proteases that are characterized by the presence of a trypsin-like proteolytic domain and at least one PDZ domain [21]. HtrA2, high temperature requirement $\mathrm{A} 2$, is the only known HtrA family member expressed in mammalian mitochondria. The full length protein is expressed at the level of the inner mitochondrial membrane where it is subject to proteolytic maturation. Its soluble form is released into the intermembrane space, where it exerts an important role in mitochondrial homeostasis and protects from neurodegeneration [22]. In the course of apoptosis, when outer mitochondrial membrane becomes permeable, Htra2 is released from the mitochondrial intermembrane space to the cytosol, where, in contrast, it exerts pro-apoptotic effects by cleaving IAPs, inhibitors of apoptosis proteins [2325]. Mnd2 mice, which carry a spontaneously occurring missense mutation in Htra2/Omi impairing the protease catalytic activity, are affected by a lethal multisystemic phenotype characterized by severe subcortical neurodegeneration. The mice display increased apoptosis particularly affecting striatal neurons, muscle wasting, and immune organ atrophy leading to death before the age of 6 weeks [22,26], all of which are similarly detected in $\mathrm{HtrA}^{-/}$mice carrying a targeted deletion in HtrA2 [27]. Interestingly, a similar phenotype was recapitulated by a neuronal specific deletion of HtrA2 [28]. Increased susceptibility to pro-apoptotic stimuli was reported in cultured cells carrying HtrA2 deletions or mutations [27,29,30], as well as reduced calcium retaining capacity [29], defective mitochondrial respiration, increased reactive oxygen species production [30,31], higher amount of mitochondrial proteins insoluble in mild detergents 
[30], and increased OPA1 processing [28], suggesting that HTRA2 is a critical protease for the maintenance of mitochondrial quality control, but providing relative little further insight in the biochemical mechanism.

The mouse wasting and immunological lethal phenotype, as well as the increased susceptibility to cell death inducers show similarities with those reported in $\mathrm{Parl}^{-/}$mice, but the first evidence that Htra2 could be a substrate of Rhomboid 7 came from work in Drosophila as discussed above [14].

Again, the identification of HTRA2 as a PARL substrate in mammals is more controversial. Starting from the above described partial phenotypical similarities between $\mathrm{Haxl}^{-/}, \mathrm{Htra2}^{-/}$, and $\mathrm{Parl}^{-/}$mice, the question was asked whether the three encoded proteins interact biochemically and functionally. Mouse fibroblasts, lymphocytes and neurons were used as model and it was investigated how the three proteins influence each other's activities in apoptosis [32]. Hax1 is a Bcl-related protein with an anti-apoptotic activity, and, like Parl, was localized in this study to mitochondrial membranes. Hax 1 was found to bind directly HtrA2 in different assays [32], and this was proposed to be needed to process HtrA2 into its intermembrane space soluble form. Levels of processed HtrA2 were decreased in Parl deficient fibroblasts. The authors found that Parl binds to Hax 1 but not to HtrA2, and therefore propose that Htra2 interacts with Parl via Hax1. They also suggest that this would explain the similarities in phenotypes in these different knock out mice. However the evidence in the paper remains circumstantial. Most importantly, a consecutive study raised indeed questions with regard to the proposed physical interactions between Hax 1 and Parl, since Hax 1 was found to be artefactually located in mitochondrial crude fractions composed by heavy membrane, but absent in purified isolated mitochondria, indicating that Hax 1 is not a mitochondrial protein [33]. An in vivo study, investigating the role or Parl and Htra2 in mouse striatal neuronal injury after transient global brain ischemia, found a parallel decrease in neuronal Parl expression and processed Htra2 levels, again supporting, although not proving, the possibility that Htra2 could be a pathophysiological relevant Parl substrate in these stress conditions [34]. Parl down regulation through siRNA significantly worsened the outcome of the transient ischemia [34]. Therefore, the question whether PARL interacts with HTRA2 independently from HAX1 remains open.

\subsection{PINK1}

PINK1 is a mitochondrial kinase with a key role in regulating mitophagy, which is expressed in low amounts at the inner membrane of healthy mitochondria. When the electrochemical mitochondrial potential becomes disrupted, PINK1 is no longer imported and it accumulates inserted at the outer membrane of the mitochondrion, phosphorylating PARKIN in the ubiquitin-like domain at position Ser65 [35-38], as well as UBIQUITIN itself also at Ser65, which triggers mitochondrial PARKIN recruitment [38-43]. This sets off a pathway involving recruitment of the E3 ubiquitin ligase PARKIN, ubiquitination of outer mitochondrial membrane proteins, and selective autophagic engulfment of the defective organelles [44-46]. This process is thought to dispose of dysfunctional mitochondria, assuring autophagy-mediated mitochondrial 
quality control. According to several authors, defective mitophagy is a major process in the pathogenesis of PINK1-related Parkinson's disease. In addition, PARKIN and PINK1 seem to be involved in another form of mitochondrial quality control mechanism, independent from macroautophagy, through disposal of small vesicular structures containing oxidized proteins and lipids, called mitochondrial derived vesicles $[47,48]$

The first evidence that PINK1 was a mitochondrial rhomboid substrate came from the already cited work by Withworth et al. [14] showing that rhomboid-7 deficient flies have impaired Pink1 processing. Soon after, several studies confirmed this in mammalian cells. Large mitochondrial accumulation of PINK1 is observed upon down regulation or genetic activation of PARL [44,49-51]. Using RNA interference, three other mitochondrial proteases were also found to affect levels of full length PINK1, including mitochondrial processing peptidase (MPP), the AFG3L2 subunit of the m-AAA protease, and ClpXP [51]. The contribution of each of these cleavages to the function of PINK1 needs further work but they might be involved in compensatory mechanisms in PARL knock out cells. In functional mitochondria with a preserved electrochemical potential, full length $63 \mathrm{kDa}$ PINK1 is imported inside mitochondria through the outer membrane translocase TOM and inner membrane translocase TIM complexes. In the matrix the mitochondrial targeting signal is removed by MPP [51], and the remaining $60 \mathrm{kDa}$ protein is inserted in the inner mitochondrial membrane. This protein is rapidly cleaved in the transmembrane domain by PARL at position Ala-103-Phe-104 to generate a $53 \mathrm{kDa}$ form [50], which rapidly retrotranslocates into the cytosol where it is degraded by the proteasome in agreement with the $\mathrm{N}$-end rule by E3 ubiquitin ligases UBRs with a fast turnover [52]. This whole cycle explains why PINK1 steady states levels are very low in healthy cells (Figure 1A). In depolarized mitochondria, import of PINK1 is inhibited and the full length form accumulates at the outer membrane (Figure 1B) [44] where it exerts ubiquitin phosphorylation as discussed. In conclusion, strong genetic and biochemical evidences support PINK1 as a real PARL substrate, although if the function of PARL-induced PINK1 cleavage is limited to regulate its turnover is still not understood, and the consequences of PARL deletion on PINK1 processing in vivo are actually unknown. Thus, amajor question remains why under normal conditions such a complicated processing of PINK1 is maintained. It would be reasonable to hypothesize that this expensive cycle of synthesis, mitochondrial transport and degradation is not only there to control mitophagy, which is not readily occurring in normal cells under non stressful conditions. One is tempted to speculate that the different steps of this process could also serve the maintenance of a not yet understood purpose. It looks like PINK1 probes the different compartments of the mitochondrion during its journey, and we hypothesize that the successful fulfillment of this journey with final dispensing of the protein might signal to the cell that mitochondria are in check. It is clear that PARL cleavage plays a pivotal role in the transformation of the membrane bound, mitophagy induction competent protein, to a soluble kinase whose function remains unfortunately still unknown at this moment. 


\subsection{PGAM5}

PGAM5 is a member of the phosphoglyceromutase enzymes which are involved in the conversion of 3-phosphoglycerate to 2-phosphoglycerate in glycolysis. PGAM5 stands apart in the family because it has a mitochondrial localization signal and lost its mutase activity during evolution. In contrast, it acquired Ser/Thr phosphatase activity [53]. PGAM5 was originally reported as an interactor of the oxidative stress sensor KEAP1 [54]. The first evidence of PGAM5 being a substrate of PARL came from Sekine et al. [55]. In contrast to the assumption that PGAM5 is a resident protein of the outer mitochondrial membrane [56], the protein was later recovered from the inner mitochondrial membrane. It was also found that it was proteolytically processed and that this increased when the mitochondrial potential collapsed, which is the opposite of PINK1 [55]. The authors also found that the processing of PGAM5 could be inhibited with dichloroisocoumarin, a serine protease inhibitor that was shown before to inhibit rhomboids [57]. This led them to test the effects of PARL down regulation, which resulted in a clear PGAM5 processing abnormality characterized by accumulation of the unprocessed form with a paralleldecrease of the processed protein. PARL down regulation also blocked the increase in PGAM5 processing after mitochondrial depolarization with mitochondria uncoupling drugs. Moreover overexpression of PARL, but not catalytically inactive PARL, induced a remarkable increase in the levels of processed PGAM5. Interestingly, after the addition of uncoupling agents to cultured cells, the authors noticed that the progressive increase in the processing of PGAM5 paralleled the increase in levels of PINK1. Then, they could show that the interactions between PARL and the two substrates PINK1 and PGAM5 were inversely correlated and that, in polarized mitochondria, PARL preferentially cleaves PINK1, while after mitochondrial depolarization PARL preferentially cleaves PGAM5, which suggests that PGAM5 processing might have a role in mitochondrial stress response. In conclusion, strong biochemical evidence supports PGAM5 as a genuine PARL substrate. However, the precise physiological functions of PGAM5 are not clear and therefore the significance of this processing step remains unclear as well. It is very intriguing that PINK1, a kinase, and PGAM5, a phosphatase, are regulated in opposite ways by PARL. We speculate that they could regulate shared substrates, although there is no evidence supporting this idea at the moment. Phosphoproteomics experiments on Pink $1^{-/-}$and Pgam $5^{-/-}$could be used to evaluate this hypothesis. Another outstanding question is to what extent PARL processing of these substrates is physiologically important. One approach could involve crossings of the different available mouse strains and check whether Parl, Pink1, and Pgam5 interact genetically and modify the severe phenotype in $\mathrm{Parl}^{-/-}$mice.

\section{Putative functions of PARL}

While the functions of the mitochondrial rhomboid has been thoroughly dissected by several independent studies in yeast $[2,7,8,58,59]$, for vertebrates the studies are more scanty. As a result, 
our understanding of PARL functions remain limited, and sometime the available data are contradictory or indirectly deduced from the available knowledge about their substrates.

\subsection{Role of PARL in apoptosis}

The wasting phenotype of the $\mathrm{Parl}^{-/}$mouse was attributed to increased apoptosis rates [11]. This was ascribed to the faster cytochrome $\mathrm{c}$ release seen in cell cultures after treatment with several intrinsic pro-apoptotic stimuli, such as staurosporine, hydrogen peroxide, etoposide, but not with the extrinsic stimulator TNF- $\alpha$. An increase in apoptotic markers was also reported in the striatum of mice after treatment with siRNA against Parl and transient global ischemia [34]. We discussed above already potential mechanisms and substrates linking PARL to apoptosis. Soluble OPA1 and HTRA2 are obvious candidates [11,32], although their status as direct PARL substrates is less clear. PGAM5 is a well-documented PARL substrate (see above paragraph), and it has been implicated in different modalities of cell death including apoptosis [53,60], and necroptosis [61], but it remains to be shown if abnormal processing of PGAM5 is necessary and sufficient to cause apoptosis in the mice. Importantly it is not excluded and actually likely that also other mechanisms contribute to the wasting phenotype in the $\mathrm{Parl}^{-/}$mice. The in vivo evidence for increased apoptosis in the $\mathrm{Parl}^{-/}$mouse was indeed largely limited to immune organs. Altogether, while the data clearly indicate a critical role for PARL in protecting cells from cell death in vitro and in vivo, the cause of the reduced life span and of the cachectic phenotype of the $\mathrm{Parl}^{--}$mouse remains to be further investigated.

\subsection{Role of PARL in shaping mitochondrial morphology}

Mitochondria constantly fuse and divide with each other, exchanging membranes, proteins, solutes, and mitochondrial DNA, to dynamically adapt within time and space to local metabolic needs, facilitate mitochondrial transport, ascertain quality control of the organelle through complementation or segregation of the damaged ones from the mitochondrial network. Heavily damaged mitochondria are eliminated through mitophagy. We discussed the effects of loss of function of $R B D 1 / P C P 1$ and $r h o-7$ on mitochondrial shape. Mitochondrial fusion rates and steady state mitochondrial network morphology was found to be normal in fibroblasts derived from the $\mathrm{Parl}^{--}$mouse [11]. However, in another study changes in mitochondrial cristae architecture were mentioned in skeletal muscle of mice locally injected with Adenoviral expressed Parl-siRNA [62] and similar mitochondrial morphological defects were detected in PARL downregulated cultured human myotubes.

The situation might be quite complicated as a shorter version of PARL, called PACT (standing for PARL $\mathrm{C}$ terminus), was found to induce mitochondrial fragmentation in cell cultures [63]. These authors propose that PARL is subject to two consecutive processing events; a first one is constitutive and occurs at position AA 52-53 to remove the mitochondrial targeting sequence, resulting in the generation of MAMP, mature mitochondrial PARL. A second, socalled, $\beta$-cleavage, is developmentally regulated and cell type specific [3]. It occurs in the matrix oriented $\mathrm{N}$ terminus domain of PARL at position AA 77-78, producing a 25 amino acid residue 
long peptide which was, surprisingly, localized into the nucleus, and the remaining $33.5 \mathrm{kDa}$ mitochondrial resident protein, PACT [3]. The $\beta$ cleavage site is only conserved in mammals, suggesting a species specific differentiation of function during evolution. Interestingly PACT was only found in cells overexpressing WT PARL but not in cells expressing catalytically inactive PARL mutants, suggesting that $\beta$ cleavage is executed by another protease activated by PARL or by PARL itself. A follow-up study from the same group identified three phosphorylation sites, at Ser-65, Thr-69, and Ser-70, with a critical role in regulating PARL $\beta$ cleavage, and this cleavage was found to be required to induce mitochondrial fragmentation [63]. It should be pointed out that all these experiments were performed in vitro in overexpressing cells, and the physiological significance of this process needs further investigation in vivo. This work suggests however complicated biochemical regulations of PARL function. Altogether, while the mitochondrial rhomboid plays indeed a role in mitochondrial shape in yeast and Drosophila, it is not clear if this also happens in mammals. Thus, more work is needed to clarify the role of PARL in modulating mitochondrial morphology and ultrastructure in vivo in mammals, as well as endogenous PARL regulation.

\subsection{Role of PARL in mitochondrial function regulation and biogenesis}

The characterization at the molecular and biochemical level of the functional consequences of Parl ablation on mitochondrial function is extremely limited. Mitochondrial respiration as well as the electrochemical mitochondrial potential appeared normal in cultured cells and muscle and liver tissues [11]. This led to the conclusion that under steady state conditions, Parl had no direct effect on the electron transport chain and supported the idea that the wasting phenotype in the mice was caused by unchecked apoptosis. This seems contradictory to what was found in another study, linking PARL deficiency to insulin resistance in diabetic patients through mitochondrial dysfunction and reduced mitochondrial mass [62]. PARL mRNA was reduced in muscle from patients with insulin resistance and type 2 diabetes, and PARL protein expression levels were reduced in primary myotubes from both type 2 diabetes patients and elderly individuals. Parl down regulation in skeletal muscle through intramuscular injection of Adenoviral PARL siRNA decreased mitochondrial mass through reduced Pgc1 $\alpha$ expression and also lowered Opa1 levels. A similar mitochondrial dysfunction was found in primary human muscle cells treated with adenoviral expressed siRNA against PARL: mitochondrial mass, mitochondrial DNA, OPA1 levels, as well as oxygen consumption, cytochrome c oxidase activity and palmitate oxidation all decreased, while reactive oxygen species increased. Moreover, myotubes treated with the short nuclear targeting PARL $\beta$ fragment responded with increasing expression of SIRT1 and OPA1, and elevated mitochondrial biogenesis. Conversely, myoblasts overexpressing PARL showed increased oxygen consumption. The authors concluded that PARL plays an important role in regulating mitochondrial mass levels by stimulating mitochondrial biogenesis through the nuclear targeting PARL $\beta$ peptide, and mitochondrial function through full length PARL, thus preventing the development of reduced insulin sensitivity. Interestingly, polymorphisms in the PARL gene detected upon a genetic screen of 1086 individuals were found 
to correlate significantly with mitochondrial DNA levels in peripheral blood cells [64]. If PARL was indeed implicated in modulating mitochondrial function and biogenesis, impairment of mitochondrial function and severely decreased mitochondrial mass would be expected in tissues of the $\mathrm{Parl}^{-/}$mouse.

In conclusion, the involvement of PARL in regulating mitochondrial function and mass deserves further investigation, including mitochondrial bioenergetics and mitochondrial ultrastructural analysis.

\subsection{Role of PARL in modulating mitophagy}

Robust evidence indicate that both PINK1 and PGAM5, two proteins involved in mitochondrial autophagic turnover, are genuine substrates of mammalian PARL [44,49$51,55,65,66]$ at least in vitro. Depolarization of mitochondria prevents processing of Pink1 by Parl because Pink1 gets stuck at the outer membrane. In one study, Parl KO MEFs transfected with PINK1-YFP, were found to have unchanged Parkin recruitment after depolarization of mitochondria with CCCP [44]. Another study reported a comparable PARKIN translocation to mitochondria in WT and PARL KO HEK293T cells [51]. At difference with these two studies, Parl KO MEFs turned out to recruit less efficiently Parkin to mitochondria in an third study [65]. Reintroduction of WT PARL, but not catalytically inactive mutant PARL, was able to rescue this biochemical phenotype. A fourth scenario was described in a study employing a doxycyclineinduced construct to down regulate PARL in HEK293T cells. Here PINK1 was found to be retrotranslocated from the inner mitochondrial membrane to the outer mitochondrial membrane, leading to massive mitochondrial PARKIN recruitment and induction of mitophagy even in the absence of mitochondrial depolarizing agents [67].

In conclusion, PARL is likely involved in mitophagy regulation, at least indirectly, as suggested by identification of PINK1 and PGAM5 as substrates, but the mechanism remains puzzling as illustrated by the very opposing results found in different experimental paradigms. One drawback is that the study of the involvement of PARL in this pathway is mostly limited to in vitro studies, often complicated by the use of protein overexpression and the prolonged use of very high concentrations of mitochondrial uncoupling drugs, which not only completely abolish the mitochondrial potential of the whole mitochondrial network in a very unphysiological manner, but also leads to a number of biochemical side effects such as enhanced ATP consumption, as a consequence of the ATP hydrolysis caused by reverse catalytic activity of ATP synthase. Moreover, the mitochondrial sublocalization of PINK1 and PGAM5 remains also controversial, with reports placing these substrates in both the mitochondrial outer membrane and in the inner membrane, as well as in the cytosol. It is possible that different pools exist for both proteins, but a more dynamic view as explained above is probably closer to the real situation. Finally, it will be important to understand the effects of the cleaved fragments versus the uncleaved PINK1 and PGAM5, as well as of any additional substrate that might become identified in the future.

\section{A possible role of PARL in diabetes and Parkinson disease}


Type 2 diabetes is a pandemic disease, resulting in a huge health care burden because of its severe systemic complications at the level of the vascular system, the eye, and renal function. The implication of mitochondrial impairment, caused by decreased mitochondrial mass, impaired oxidative phosphorylation capacity, and increased reactive oxygen species production has been suggested by several studies.

Gene variants in the PARL gene were first reported as a putative risk factor for type 2 diabetes in a gene expression study in skeletal muscle aimed to identify differentially expressed mitochondrial genes in the Psammomys obesus, a rodent susceptible to metabolic diseases including obesity and type 2 diabetes [68]. Parl gene expression was significantly reduced in the diabetic rodents, while this deficit improved after exercise training. A population of 1031 human North American subjects was screened and a polymorphism Leu262Val was found in PARL which was associated with increased plasma insulin concentrations, a known risk factor for diabetes. However, another study conducted on a sample of 3666 individuals, failed to detect this association, and concluded that this genetic variant was unlikely to be an important contributor to insulin resistance in the UK population [69]. A third study, conducted on a sample of 613 Irish individuals also failed to identify the association between this PARL gene variant and plasma insulin, but found associations between homozygous carriers of this variant and microalbuminuria, a marker of diabetic nephropathy, as well as with the occurrence of earlier onset of type 2 diabetes [70]. Decreased PARL protein and gene expression, and lower mitochondrial mass were found in both skeletal muscle biopsies and cultured myotubes from elderly individuals and patients with type 2 diabetes compared to controls [62]. Down regulation of PARL in muscle, as discussed above, caused increased triglyceride content and impaired insulin signaling. High fat diet increased insulin resistance and decreased Parl expression in mice. The authors concluded that PARL expression may contribute to the mitochondrial abnormalities found in ageing and type 2 diabetes [62]. Altogether, while the genetic variations in the PARL gene are likely very rare and modest risk factors for the development of type 2 diabetes, PARL may be an interesting candidate to study in further depth the mitochondrial pathophysiology at the basis of this disease.

Parkinson's disease (PD) is the second most prevalent neurodegenerative disease, leading to severe disability and huge health care impact. The first link between PD and PARL was made when Pink1 was identified as a substrate of Rhomboid-7 in Drosophila [14]. Following studies confirmed that PINK1 was a substrate of the mitochondrial rhomboid in mammals [44,49$51,65]$. Interestingly, a missense mutation, c.230G $>A$, was found in the PARL gene in two patients affected by PD [65]. This mutation results in a substitution of a serine to an asparagine at codon 77 (p.Ser77Asn) and is placed in a highly conserved phosphorylation site in the PARL N terminus with a critical role in regulation of PARL $\beta$ cleavage discussed above $[3,63,65]$. However, two independent studies, one in 92 patients affected by early onset PD [71], another in more than 2000 German patients including both sporadic and familial cases [72], failed to detect 
the presence of c.230G >A or of other pathogenic mutations in the PARL gene, suggesting that PARL mutations are an extremely rare genetic cause of PD.

Interestingly, another link between PD and PARL was indirectly provided by the description of a late onset Parkinsonian phenotype in the $\operatorname{Pgam5}^{-1-}$ mouse [66]. These mice were affected by dopaminergic neurodegeneration in the substantia nigra pars compacta at 1 year of age, and show decreased dopamine and dopamine metabolites, and locomotor impairment which improved by L-DOPA therapy [66]. However, other reports from independent groups reported different phenotypes in the $P g a m 5^{-/}$mouse, such as decreased body weight, reduced survival in males, and impaired inflammasome activation [73], and increased resistance to combined cold stress and fasting, as well as resistance to high-fat-diet-induced obesity [74].

Altogether, the available evidence indicates at least an indirect role of PARL in PD pathophysiology through its substrate PINK1, and potentially PGAM5, although how their cleavage by PARL might possibly affect the development of PD is not yet understood. Variants in the PARL gene seems to be very rare causes of $\mathrm{PD}$, but genetic screenings of the whole $P A R L$ gene exons on larger samples of patients with PD from different ethnic groups are warranted.

\section{Conclusions}

The discovery of a mitochondria-localized rhomboid more than one decade ago opened a new area of investigation in mitochondrial biology and brought the field of regulated intra-membrane proteolysis also inside the mitochondria. The presence of severe phenotypes in all species where the mitochondrial rhomboid has been genetically ablated unequivocally indicates a crucial physiological role of this rhomboid in cell homeostasis and the absence of redundant biochemical pathways leading to effective compensation. Pioneering studies have defined a limited number of substrates and a role in regulating different critical aspects of mitochondrial biology, including mitochondrial morphology, apoptosis, mitophagy, mitochondrial metabolism, and in the pathophysiology of prevalent diseases such as diabetes and Parkinson's disease. Although several of these reports, especially in mammals, are preliminary and often contradictory, and many observations are limited to in vitro analysis, often further confounded by protein overexpression, the available evidence clearly indicates a fundamental role of PARL in mitochondrial biology. Additional studies are warranted to confirm the proposed PARL substrates in vivo, investigate the presence of additional substrates, and define their significance in physiology and in human diseases. Several approaches could be used to identify novel mitochondrial rhomboid substrates and interactors, including coimmunoprecipitation, copurification using affinity tags, cross-linking experiments, or mass spectrometry based degradome analysis. A careful reevaluation of the physiological mitochondrial functions of PARL in different animal species is also needed in order to define more precisely which biological functions are conserved during evolution. Finally we 
propose to associate the $\mathrm{P}$ in the name PARL no longer to Presenilin, but to the known substrates PGAM5 and PINK1, which could help to make the literature on these intriguing proteins less confusing.

\section{Legend to figure}

Schematic cartoon illustrating PARL, the mitochondrial rhomboid located in the inner mitochondrial membrane (IMM), and its putative interacting proteins. (A) In polarized mitochondria with preserved mitochondrial membrane potential $(\Delta \psi)$, PARL preferentially cleaves PINK1. PINK1 is normally imported through the TOM/TIM complexes, its mitochondrial targeting signaling is cleaved by matrix MPP, it is inserted in the inner mitochondrial membrane through its transmembrane domain, at which level it is cleaved by PARL. Then, through still unknown mechanisms, it is thought to be rerouted to the cytosol, where it is rapidly degraded by the ubiquitin-proteasome system. Although still under debate, PGAM5 could potentially have a double mitochondrial sublocalization, an inner membrane pool, which is only marginally cleaved by PARL in polarized mitochondria, and possibly an outer membrane pool which may possibly interact with KEAP1 and the antioxidant response regulator NRF2 [56]. It is possible, but controversial, that PARL also cleaves OPA1, generating a shorter intermembrane space (IMS) OPA1 pool which serves keeping mitochondrial cristae junction tight, delaying cristae remodeling during apoptosis [11], as well as the peptidase HTRA2 [32],

generating its mature intermembrane space resident from, which protects from neurodegeneration. (B) In depolarized mitochondria with collapsed mitochondrial potential $(\Delta \psi)$, PINK1 is no longer fully imported inside the mitochondrial matrix passing through TOM and TIM complexes and it is inserted in the mitochondrial outer membrane (OMM) (directly, or indirectly through rerouting to the outer membrane), through its transmembrane domain, leading to PARKIN recruitment from the cytosol to the mitochondria and activating mitophagy, which eliminates dysfunctional mitochondria. In these stress conditions, PARL cleaves PGAM5 generating a 24 aminoacids shorter form [55] of which both function and mitochondrial sublocalization are not known. A putative pool of PGAM5 residing in the outer mitochondrial membrane membrane has been reported to dephosphorylate FUNDC1, activating mitophagy [75]. In particular conditions causing necroptosis induction, requiring high RIP3 expression and caspase 8 inhibition, and only in specific cell types, the putative outer membrane pool of PGAM5 has been reported as a component of the necrosome complex responsible for dephosphorylation and activation of the mitochondrial fission protein DRP1 [61], although following studies have shown PGAM5 is dispensable for necroptosis execution, as discussed above.

\section{Acknowledgments}

This work was supported by the European Research Council (ERC) Grant ERC-2010AG_268675 to BDS, the Fonds voor Wetenschappelijk Onderzoek (FWO), Stichting Alzheimer 
Onderzoek, the KU Leuven and VIB, a Methusalem grant of the KU Leuven/Flemish Government to BDS, Interuniversitary attraction pool IAP P7-16, and Instituut voor Wetenschappen en Technologie project IWT-VIND 135043.

MS is recipient of an EMBO Long-term fellow (ALTF 648-2013). BDS is supported by the BaxVanluffelen Chair for Alzheimer's Disease and "Opening the Future" of the Leuven Universiteit Fonds (LUF). BDS is a consultant for Janssen Pharmaceutica, Beerse; Forum Pharmaceuticals, Boston; and Remynd NV, Leuven.

\section{References}

[1] L. Pellegrini, B.J. Passer, M. Canelles, I. Lefterov, J.K. Ganjei, B.J. Fowlkes, E. V. Koonin, L. D'Adamio, PAMP and PARL, two novel putative metalloproteases interacting with the COOH-terminus of Presenilin-1 and -2., J. Alzheimers. Dis. 3 (2001) 181-190.

[2] G.A. McQuibban, S. Saurya, M. Freeman, Mitochondrial membrane remodelling regulated by a conserved rhomboid protease, Nature. 423 (2003) 537-541.

[3] A. S??k, B.J. Passer, E. V. Koonin, L. Pellegrini, Self-regulated Cleavage of the Mitochondrial Intramembrane-cleaving Protease PARL Yields P??, a Nuclear-targeted Peptide, J. Biol. Chem. 279 (2004) 15323-15329.

[4] D. V Jeyaraju, H.M. McBride, R.B. Hill, L. Pellegrini, Structural and mechanistic basis of Parl activity and regulation., Cell Death Differ. 18 (2011) 1531-1539.

[5] K. Esser, B. Tursun, M. Ingenhoven, G. Michaelis, E. Pratje, A novel two-step mechanism for removal of a mitochondrial signal sequence involves the mAAA complex and the putative rhomboid protease Pcp1, J. Mol. Biol. 323 (2002) 835-843.

[6] G. Michaelis, K. Esser, B. Tursun, J.P. Stohn, S. Hanson, E. Pratje, Mitochondrial signal peptidases of yeast: The rhomboid peptidase Pcp1 and its substrate cytochrome $\mathrm{c}$ peroxidase, Gene. 354 (2005) 58-63.

[7] T. Tatsuta, S. Augustin, M. Nolden, B. Friedrichs, T. Langer, m-AAA protease-driven membrane dislocation allows intramembrane cleavage by rhomboid in mitochondria., EMBO J. 26 (2007) 325-335.

[8] M. Herlan, F. Vogel, C. Bornhövd, W. Neupert, A.S. Reichert, Processing of Mgm1 by the rhomboid-type protease Pcpl is required for maintenance of mitochondrial morphology and of mitochondrial DNA, J. Biol. Chem. 278 (2003) 27781-27788.

[9] G.A. McQuibban, J.R. Lee, L. Zheng, M. Juusola, M. Freeman, Normal Mitochondrial Dynamics Requires Rhomboid-7 and Affects Drosophila Lifespan and Neuronal Function, Curr. Biol. 16 (2006) 982-989.

[10] M. Rahman, P. Kylsten, Rhomboid-7 over-expression results in Opa1-like processing and malfunctioning mitochondria, Biochem. Biophys. Res. Commun. 414 (2011) 315-320.

[11] S. Cipolat, T. Rudka, D. Hartmann, V. Costa, L. Serneels, K. Craessaerts, K. Metzger, C. Frezza, W. Annaert, L. D'Adamio, C. Derks, T. Dejaegere, L. Pellegrini, R. D’Hooge, L. Scorrano, B. De Strooper, Mitochondrial Rhomboid PARL Regulates Cytochrome c Release during Apoptosis via OPA1-Dependent Cristae Remodeling, Cell. 126 (2006) 163-175.

[12] I.E. Clark, M.W. Dodson, C. Jiang, J.H. Cao, J.R. Huh, J.H. Seol, S.J. Yoo, B. a Hay, M. 
Guo, Drosophila pink1 is required for mitochondrial function and interacts genetically with parkin., Nature. 441 (2006) 1162-1166.

[13] J. Park, S.B. Lee, S.B. Lee, Y. Kim, S. Song, S. Kim, E. Bae, J.M. Kim, M.H. Shong, J.M. Kim, J.K. Chung, Mitochondrial dysfunction in Drosophila PINK1 mutants is complemented by parkin, Nature. 441 (2006) 1157-1161.

[14] A.J. Whitworth, J.R. Lee, V.M.-W. Ho, R. Flick, R. Chowdhury, G.A. McQuibban, Rhomboid-7 and HtrA2/Omi act in a common pathway with the Parkinson's disease factors Pink1 and Parkin, Dis. Model. Mech. 1 (2008) 168-174.

[15] S. Noble, A. Ismail, R. Godoy, Y. Xi, M. Ekker, Zebrafish Parla- and Parlb-deficiency affects dopaminergic neuron patterning and embryonic survival, J. Neurochem. 122 (2012) 196-207.

[16] N. Ishihara, Y. Fujita, T. Oka, K. Mihara, Regulation of mitochondrial morphology through proteolytic cleavage of OPA1., EMBO J. 25 (2006) 2966-2977.

[17] S. Duvezin-Caubet, M. Koppen, J. Wagener, M. Zick, L. Israel, A. Bernacchia, R. Jagasia, E.I. Rugarli, A. Imhof, W. Neupert, T. Langer, A.S. Reichert, OPA1 processing reconstituted in yeast depends on the subunit composition of the m-AAA protease in mitochondria., Mol. Biol. Cell. 18 (2007) 3582-90.

[18] S. Ehses, I. Raschke, G. Mancuso, A. Bernacchia, S. Geimer, D. Tondera, J.C. Martinou, B. Westermann, E.I. Rugarli, T. Langer, Regulation of OPA1 processing and mitochondrial fusion by m-AAA protease isoenzymes and OMA1, J. Cell Biol. 187 (2009) 1023-1036.

[19] L. Griparic, T. Kanazawa, A.M. Van Der Bliek, Regulation of the mitochondrial dynaminlike protein Opa1 by proteolytic cleavage, J. Cell Biol. 178 (2007) 757-764.

[20] O. Guillery, F. Malka, T. Landes, E. Guillou, C. Blackstone, A. Lombès, P. Belenguer, D. Arnoult, M. Rojo, Metalloprotease-mediated OPA1 processing is modulated by the mitochondrial membrane potential., Biol. Cell. 100 (2008) 315-25.

[21] L. Vande Walle, M. Lamkanfi, P. Vandenabeele, The mitochondrial serine protease HtrA2/Omi: an overview., Cell Death Differ. 15 (2008) 453-460.

[22] S. Rathke-Hartlieb, U. Schlomann, P. Heimann, M.H. Meisler, H. Jockusch, J.W. Bartsch, Progressive loss of striatal neurons causes motor dysfunction in MND2 mutant mice and is not prevented by Bcl-2., Exp. Neurol. 175 (2002) 87-97.

[23] Q.H. Yang, R. Church-Hajduk, J. Ren, M.L. Newton, C. Du, Omi/HtrA2 catalytic cleavage of inhibitor of apoptosis (IAP) irreversibly inactivates IAPs and facilitates caspase activity in apoptosis, Genes Dev. 17 (2003) 1487-1496.

[24] Y. Suzuki, Y. Imai, H. Nakayama, K. Takahashi, K. Takio, R. Takahashi, A serine protease, HtrA2, is released from the mitochondria and interacts with XIAP, inducing cell death, Mol. Cell. 8 (2001) 613-621.

[25] S.M. Srinivasula, S. Gupta, P. Datta, Z. Zhang, R. Hegde, N. Cheong, T. FernandesAlnemri, E.S. Alnemri, Inhibitor of apoptosis proteins are substrates for the mitochondrial serine protease Omi/HtrA2, J. Biol. Chem. 278 (2003) 31469-31472.

[26] J.M. Jones, R.L. Albin, E.L. Feldman, K. Simin, T.G. Schuster, W.A. Dunnick, J.T. Collins, C.E. Chrisp, B.A. Taylor, M.H. Meisler, Mnd2: a New Mouse Model of Inherited Motor Neuron Disease., Genomics. 16 (1993) 669-77.

[27] L.M. Martins, A. Morrison, K. Klupsch, V. Fedele, N. Moisoi, P. Teismann, A. Abuin, E. Grau, M. Geppert, P. George, C.L. Creasy, A. Martin, I. Hargreaves, S.J. Heales, H. Okada, S. Brandner, B. Schulz, T. Mak, J. Downward, G.P. Livi, Neuroprotective Role of 
the Reaper-Related Serine Protease HtrA2 / Omi Revealed by Targeted Deletion in Mice Neuroprotective Role of the Reaper-Related Serine Protease HtrA2 / Omi Revealed by Targeted Deletion in Mice, Mol. Cell. Biol. 24 (2004) 9848-9862.

[28] V.L. Patterson, A.J. Zullo, C. Koenig, S. Stoessel, H. Jo, X. Liu, J. Han, M. Choi, A.T. DeWan, J.L. Thomas, C.Y. Kuan, J. Hoh, Neural-specific deletion of HTRA2 causes cerebellar neurodegeneration and defective processing of mitochondrial OPA1, PLoS One. 9 (2014) 1-24.

[29] J.M. Jones, P. Datta, S.M. Srinivasula, W. Ji, S. Gupta, Z. Zhang, E. Davies, G. Hajnóczky, T.L. Saunders, M.L. Van Keuren, T. Fernandes-Alnemri, M.H. Meisler, E.S. Alnemri, Loss of Omi mitochondrial protease activity causes the neuromuscular disorder of mnd2 mutant mice., Nature. 425 (2003) 721-7.

[30] N. Moisoi, K. Klupsch, V. Fedele, P. East, S. Sharma, a Renton, H. Plun-Favreau, R.E. Edwards, P. Teismann, M.D. Esposti, a D. Morrison, N.W. Wood, J. Downward, L.M. Martins, Mitochondrial dysfunction triggered by loss of HtrA2 results in the activation of a brain-specific transcriptional stress response., Cell Death Differ. 16 (2009) 449-464.

[31] N. Kieper, K.M. Holmström, D. Ciceri, F.C. Fiesel, H. Wolburg, E. Ziviani, A.J. Whitworth, L.M. Martins, P.J. Kahle, R. Krüger, Modulation of mitochondrial function and morphology by interaction of Omi/HtrA 2 with the mitochondrial fusion factor OPA1, Exp. Cell Res. 316 (2010) 1213-1224.

[32] J.-R. Chao, E. Parganas, K. Boyd, C.Y. Hong, J.T. Opferman, J.N. Ihle, Hax 1-mediated processing of HtrA2 by Parl allows survival of lymphocytes and neurons., Nature. 452 (2008) 98-102.

[33] D. V Jeyaraju, G. Cisbani, O.M. De Brito, E. V Koonin, L. Pellegrini, Hax 1 lacks BH modules and is peripherally associated to heavy membranes: implications for Omi/HtrA2 and PARL activity in the regulation of mitochondrial stress and apoptosis., Cell Death Differ. 16 (2009) 1622-1629.

[34] H. Yoshioka, M. Katsu, H. Sakata, N. Okami, T. Wakai, H. Kinouchi, P.H. Chan, The role of PARL and HtrA2 in striatal neuronal injury after transient global cerebral ischemia., J. Cereb. Blood Flow Metab. 33 (2013) 1658-65.

[35] K. Shiba-Fukushima, Y. Imai, S. Yoshida, Y. Ishihama, T. Kanao, S. Sato, N. Hattori, PINK1-mediated phosphorylation of the Parkin ubiquitin-like domain primes mitochondrial translocation of Parkin and regulates mitophagy., Sci. Rep. 2 (2012) 1002.

[36] C. Kondapalli, A. Kazlauskaite, N. Zhang, H.I. Woodroof, D.G. Campbell, R. Gourlay, L. Burchell, H. Walden, T.J. Macartney, M. Deak, A. Knebel, D.R. Alessi, M.M.K. Muqit, PINK1 is activated by mitochondrial membrane potential depolarization and stimulates Parkin E3 ligase activity by phosphorylating Serine 65, Open Biol. 2 (2012) 120080 120080.

[37] M. Iguchi, Y. Kujuro, K. Okatsu, F. Koyano, H. Kosako, M. Kimura, N. Suzuki, S. Uchiyama, K. Tanaka, N. Matsuda, Parkin-catalyzed Ubiquitin-Ester Transfer Is Triggered by PINK1-dependent Phosphorylation, J. Biol. Chem. 288 (2013) 22019-22032.

[38] A. Kazlauskaite, C. Kondapalli, R. Gourlay, D.G. Campbell, M.S. Ritorto, K. Hofmann, D.R. Alessi, A. Knebel, M. Trost, M.M.K. Muqit, Parkin is activated by PINK1-dependent phosphorylation of ubiquitin at Ser ${ }^{65}$, Biochem. J. 460 (2014) 127-141.

[39] F. Koyano, K. Okatsu, H. Kosako, Y. Tamura, E. Go, M. Kimura, Y. Kimura, H. Tsuchiya, H. Yoshihara, T. Hirokawa, T. Endo, E. a Fon, J.-F. Trempe, Y. Saeki, K. Tanaka, N. Matsuda, Ubiquitin is phosphorylated by PINK1 to activate parkin., Nature. 
510 (2014) 162-6.

[40] L.A. Kane, M. Lazarou, A.I. Fogel, Y. Li, K. Yamano, S.A. Sarraf, S. Banerjee, R.J. Youle, PINK1 phosphorylates ubiquitin to activate parkin E3 ubiquitin ligase activity, J. Cell Biol. 205 (2014) 143-153.

[41] T. Wauer, K.N. Swatek, J.L. Wagstaff, C. Gladkova, J.N. Pruneda, M.A. Michel, M. Gersch, C.M. Johnson, S.M. V Freund, D. Komander, Ubiquitin Ser65 phosphorylation affects ubiquitin structure, chain assembly and hydrolysis., EMBO J. 34 (2014) 1-20.

[42] K. Okatsu, F. Koyano, M. Kimura, H. Kosako, Y. Saeki, K. Tanaka, N. Matsuda, Phosphorylated ubiquitin chain is the genuine Parkin receptor, J. Cell Biol. 209 (2015) 111-128.

[43] K. Shiba-Fukushima, T. Arano, G. Matsumoto, T. Inoshita, S. Yoshida, Y. Ishihama, K.Y. Ryu, N. Nukina, N. Hattori, Y. Imai, Phosphorylation of Mitochondrial Polyubiquitin by PINK1 Promotes Parkin Mitochondrial Tethering, PLoS Genet. 10 (2014).

[44] S.M. Jin, M. Lazarou, C. Wang, L.A. Kane, D.P. Narendra, R.J. Youle, Mitochondrial membrane potential regulates PINK1 import and proteolytic destabilization by PARL, J. Cell Biol. 191 (2010) 933-942.

[45] D.P. Narendra, S.M. Jin, A. Tanaka, D.F. Suen, C.A. Gautier, J. Shen, M.R. Cookson, R.J. Youle, PINK1 is selectively stabilized on impaired mitochondria to activate Parkin, PLoS Biol. 8 (2010).

[46] C. Vives-Bauza, C. Zhou, Y. Huang, M. Cui, R.L.A. de Vries, J. Kim, J. May, M.A. Tocilescu, W. Liu, H.S. Ko, J. Magrane, D.J. Moore, V.L. Dawson, R. Grailhe, T.M. Dawson, C. Li, K. Tieu, S. Przedborski, PINK1-dependent recruitment of Parkin to mitochondria in mitophagy, Proc. Natl. Acad. Sci. U. S. A. 107 (2010) 378-383.

[47] G. Mclelland, V. Soubannier, C.X. Chen, H.M. Mcbride, E.A. Fon, Parkin and PINK 1 function in a vesicular trafficking pathway regulating mitochondrial quality control, 33 (2014) 282-295.

[48] A. Sugiura, G. Mclelland, E.A. Fon, H.M. Mcbride, A new pathway for mitochondrial quality control : mitochondrial-derived vesicles, 33 (2014) 2142-2156.

[49] C. Meissner, H. Lorenz, A. Weihofen, D.J. Selkoe, M.K. Lemberg, The mitochondrial intramembrane protease PARL cleaves human Pink1 to regulate Pink1 trafficking, J. Neurochem. 117 (2011) 856-867.

[50] E. Deas, H. Plun-Favreau, S. Gandhi, H. Desmond, S. Kjaer, S.H.Y. Loh, A.E.M. Renton, R.J. Harvey, A.J. Whitworth, L.M. Martins, A.Y. Abramov, N.W. Wood, PINK1 cleavage at position A103 by the mitochondrial protease PARL, Hum. Mol. Genet. 20 (2011) 867879.

[51] A.W. Greene, K. Grenier, M. a Aguileta, S. Muise, R. Farazifard, M.E. Haque, H.M. McBride, D.S. Park, E. a Fon, Mitochondrial processing peptidase regulates PINK1 processing, import and Parkin recruitment, EMBO Rep. 13 (2012) 378-385.

[52] K. Yamano, R.J. Youle, K. Yamano, R.J. Youle, PINK1 is degraded through the N-end rule pathway PINK1 is degraded through the N-end rule pathway, 8627 (2016).

[53] K. Takeda, Y. Komuro, T. Hayakawa, H. Oguchi, Y. Ishida, S. Murakami, T. Noguchi, H. Kinoshita, Y. Sekine, S. Iemura, T. Natsume, H. Ichijo, Mitochondrial phosphoglycerate mutase 5 uses alternate catalytic activity as a protein serine/threonine phosphatase to activate ASK1., Proc. Natl. Acad. Sci. U. S. A. 106 (2009) 12301-12305.

[54] S.C. Lo, M. Hannink, PGAM5, a Bcl-XL-interacting protein, is a novel substrate for the redox-regulated Keap1-dependent ubiquitin ligase complex, J. Biol. Chem. 281 (2006) 
37893-37903.

[55] S. Sekine, Y. Kanamaru, M. Koike, A. Nishihara, M. Okada, H. Kinoshita, M. Kamiyama, J. Maruyama, Y. Uchiyama, N. Ishihara, K. Takeda, H. Ichijo, Rhomboid protease PARL mediates the mitochondrial membrane potential loss-induced cleavage of PGAM5, J. Biol. Chem. 287 (2012) 34635-34645.

[56] S.C. Lo, M. Hannink, PGAM5 tethers a ternary complex containing Keap1 and Nrf2 to mitochondria, Exp. Cell Res. 314 (2008) 1789-1803.

[57] S. Urban, M.S. Wolfe, Reconstitution of intramembrane proteolysis in vitro reveals that pure rhomboid is sufficient for catalysis and specificity., Proc. Natl. Acad. Sci. U. S. A. 102 (2005) 1883-8.

[58] H. Sesaki, S.M. Southard, A.E. Aiken Hobbs, R.E. Jensen, Cells lacking Pcp1p/Ugo2p, a rhomboid-like protease required for Mgm1p processing, lose mtDNA and mitochondrial structure in a Dnm1p-dependent manner, but remain competent for mitochondrial fusion, Biochem. Biophys. Res. Commun. 308 (2003) 276-283.

[59] A. Sch??fer, M. Zick, J. Kief, M. Steger, H. Heide, S. Duvezin-Caubet, W. Neupert, A.S. Reichert, Intramembrane proteolysis of Mgm1 by the mitochondrial rhomboid protease is highly promiscuous regarding the sequence of the cleaved hydrophobic segment, J. Mol. Biol. 401 (2010) 182-193.

[60] M. Zhuang, S. Guan, H. Wang, A.L. Burlingame, J.A. Wells, Substrates of IAP Ubiquitin Ligases Identified with a Designed Orthogonal E3 Ligase, the NEDDylator, Mol. Cell. 49 (2013) 273-282.

[61] Z. Wang, H. Jiang, S. Chen, F. Du, X. Wang, The mitochondrial phosphatase PGAM5 functions at the convergence point of multiple necrotic death pathways, Cell. 148 (2012) $228-243$.

[62] A.E. Civitarese, P.S. MacLean, S. Carling, L. Kerr-Bayles, R.P. Mcmillan, A. Pierce, T.C. Becker, C. Moro, J. Finlayson, N. Lefort, C.B. Newgard, L. Mandarino, W. Cefalu, K. Walder, G.R. Collier, M.W. Hulver, S.R. Smith, E. Ravussin, N. Brunswick, Regulation of skeletal muscle oxidative capacity and insulin signaling by the mitochondrial rhomboid protease PARL, Cell. 11 (2010) 412-426.

[63] D. V Jeyaraju, L. Xu, M.-C. Letellier, S. Bandaru, R. Zunino, E.A. Berg, H.M. Mcbride, L. Pellegrini, Phosphorylation and cleavage of presenilin-associated rhomboid-like protein (PARL) promotes changes in mitochondrial morphology, Proc. Natl. Acad. Sci. U. S. A. 103 (2006) 18562-18567.

[64] J.E. Curran, J.B.M. Jowett, L.J. Abraham, L.A. Diepeveen, K.S. Elliott, T.D. Dyer, L.J. Kerr-Bayles, M.P. Johnson, A.G. Comuzzie, E.K. Moses, K.R. Walder, G.R. Collier, J. Blangero, A.H. Kissebah, Genetic variation in PARL influences mitochondrial content, Hum. Genet. 127 (2010) 183-190.

[65] G. Shi, J.R. Lee, D.A. Grimes, L. Racacho, D. Ye, H. Yang, O.A. Ross, M. Farrer, G.A. McQuibban, D.E. Bulman, Functional alteration of PARL contributes to mitochondrial dysregulation in Parkinson's disease, Hum. Mol. Genet. 20 (2011) 1966-1974.

[66] W. Lu, S.S. Karuppagounder, D.A. Springer, M.D. Allen, L. Zheng, B. Chao, Y. Zhang, V.L. Dawson, T.M. Dawson, M. Lenardo, Genetic deficiency of the mitochondrial protein PGAM5 causes a Parkinson's-like movement disorder, Nat. Commun. 5 (2014) 4930.

[67] C. Meissner, H. Lorenz, B. Hehn, M.K. Lemberg, Intramembrane protease PARL defines a negative regulator of PINK1- and PARK2/Parkin-dependent mitophagy, Autophagy. 11 (2015) 1484-1498. 
[68] K. Walder, L. Kerr-Bayles, A. Civitarese, J. Jowett, J. Curran, K. Elliott, J. Trevaskis, N. Bishara, P. Zimmet, L. Mandarino, E. Ravussin, J. Blangero, A. Kissebah, G.R. Collier, The mitochondrial rhomboid protease PSARL is a new candidate gene for type 2 diabetes, Diabetologia. 48 (2005) 459-468.

[69] K.A. Fawcett, N.J. Wareham, J. Luan, H. Syddall, C. Cooper, S. O’Rahilly, I.N.M. Day, M.S. Sandhu, I. Barroso, PARL Leu262Val is not associated with fasting insulin levels in UK populations, Diabetologia. 49 (2006) 2649-2652.

[70] M. Hatunic, M. Stapleton, E. Hand, C. DeLong, V.E.F. Crowley, J.J. Nolan, The Leu262Val polymorphism of presenilin associated rhomboid like protein (PARL) is associated with earlier onset of type 2 diabetes and increased urinary microalbumin creatinine ratio in an Irish case-control population, Diabetes Res. Clin. Pract. 83 (2009) 316-319.

[71] S. Heinitz, C. Klein, The p.S77N presenilin-associated rhomboid-like protein mutation is not a frequent cause of early-onset Parkinson's disease, Mov. Disord. 26 (2011) 2441-2.

[72] R. Wüst, B. Maurer, K. Hauser, D. Woitalla, M. Sharma, R. Krüger, Mutation analyses and association studies to assess the role of the presenilin associated rhomboid like (PARL) gene in Parkinson's disease, Neurobiol. Aging. (2015) 1-3.

[73] K. Moriwaki, N. Farias Luz, S. Balaji, M.J. De Rosa, C.L. O’Donnell, P.J. Gough, J. Bertin, R.M. Welsh, F.K.-M. Chan, The Mitochondrial Phosphatase PGAM5 Is Dispensable for Necroptosis but Promotes Inflammasome Activation in Macrophages., J. Immunol. (2015).

[74] S. Sekine, A. Yao, K. Hattori, S. Sugawara, I. Naguro, M. Koike, Y. Uchiyama, K. Takeda, H. Ichijo, The Ablation of Mitochondrial Protein Phosphatase Pgam5 Confers Resistance Against Metabolic Stress, EBioMedicine. 5 (2016) 82-92.

[75] G. Chen, Z. Han, D. Feng, Y. Chen, L. Chen, H. Wu, L. Huang, C. Zhou, X. Cai, C. Fu, L. Duan, X. Wang, L. Liu, X. Liu, Y. Shen, Y. Zhu, Q. Chen, A regulatory signaling loop comprising the PGAM5 phosphatase and CK2 controls receptor-mediated mitophagy, Mol. Cell. 54 (2014) 362-377. 
A

$$
\text { 品品 }
$$

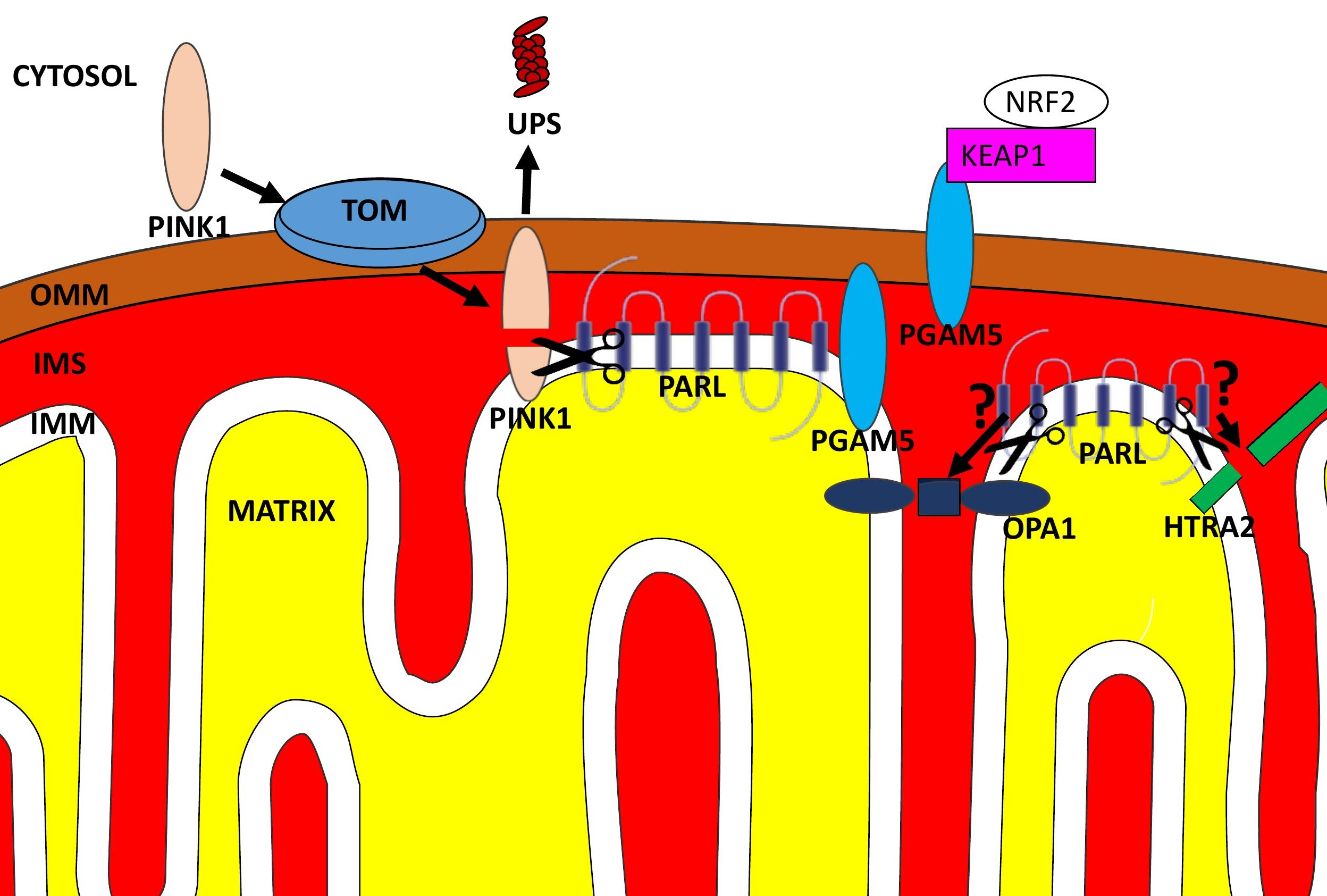




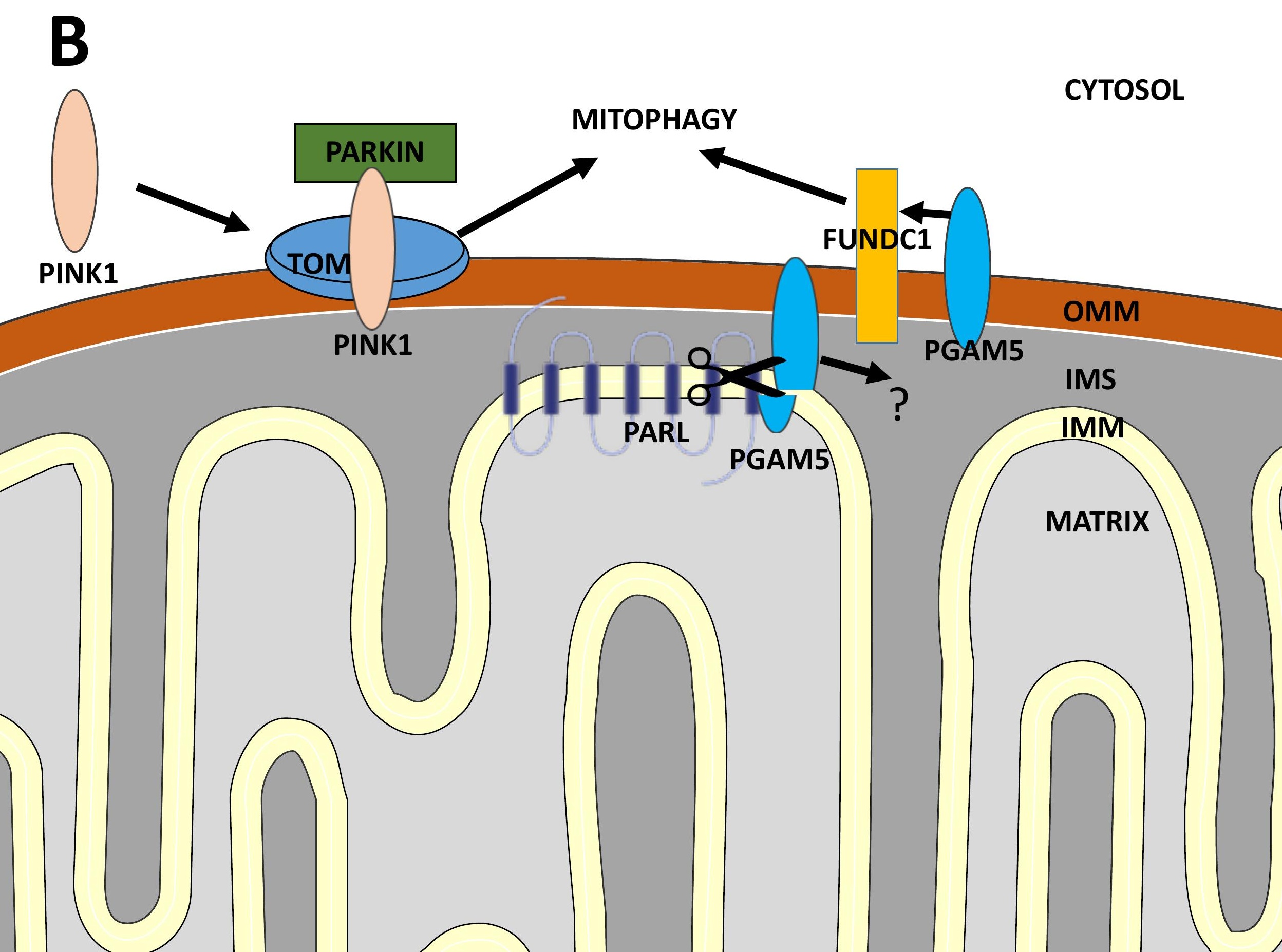

